\title{
Archéopages
}

Archéopages Archéologie et société

$46 \mid 2018$

Maisons

\section{Être chez soi. Débat}

Propos recueillis par Catherine Chauveau

Anick Coudart, Laurent Schneider, Hélène Wurmser et Catherine Chauveau

\section{(2) OpenEdition}

Journals

Édition électronique

URL : https://journals.openedition.org/archeopages/4487

DOI : $10.4000 /$ archeopages. 4487

ISSN : 2269-9872

Éditeur

INRAP - Institut national de recherches archéologiques préventives

\section{Édition imprimée}

Date de publication : 1 décembre 2018

Pagination : 86-93

ISSN : 1622-8545

Référence électronique

Anick Coudart, Laurent Schneider, Hélène Wurmser et Catherine Chauveau, «Être chez soi. Débat », Archéopages [En ligne], 46 | 2018, mis en ligne le 01 décembre 2020, consulté le 03 juin 2021. URL : http://journals.openedition.org/archeopages/4487 ; DOI : https://doi.org/10.4000/archeopages.4487 


\section{Toute modeste soit-elle, la maison est toujours plus qu'un gîte. Si celui-ci renvoie à une question purement animale - se protéger dans des conditions de vulnérabilité, face aux intempéries ou pendant le sommeil, la maison matérialise les barrières entre soi et l'autre. Mais comment distinguer ce type de construction des autres?}

\section{Débat}

\section{Anick Coudart}

86

spécialiste du Néolithique
Archéologue et ethnologue, européen et de l'habitat des Anga de Papouasie, elle a collecté des milliers de données technologiques dans le Sud-Ouest des États-Unis, au Mexique, en Mongolie, en Afrique du Sud, dans le Maghreb, en Chine et au Japon. Elle a notamment publié « Le Temps de la maison... Le Temps de la culture », in B. Latour et P. Lemonnier (dir.), De la Préhistoire aux missiles balistiques : l'intelligence sociale des techniques, Paris, La Découverte, 1994, p. 228-252; Architecture et société néolithiques, Daf 67, Paris, Éds de la MSH, 1998 ; « Maisons d'hier ou maisons d'aujourd'hui. L'espace domestique fabrique et donne à voir la société ", in F. Braemer F., S. Cleuziou et A. Coudart (dir), Habitat et Société, Antibes, Éditions APDCA, 1999, p. 536-548; « La maison néolithique : métaphore matérielle, sociale et mentale des petites sociétés sédentaires », in J.-P. Demoule (dir.), La révolution néolithique dans le monde, Paris, éditions du CNRS, 2009, p. 219-239; « The Bandkeramik longhouses », in C. Fowler, J. Harding et D. Hofmann (dir.), The Oxford Handbook of Neolithic Europe, Oxford, Oxford University Press, 2015, pp. 309-325.

\section{Laurent Schneider}

est directeur de recherches au

CNRS et directeur d'études à

l'Ehess, au Ciham (histoire, archéologie, littérature des mondes chrétiens et musulmans médiévaux). Il travaille notamment sur les dynamiques de transformation des systèmes d'habitat. Parmi ses publications : Structures du peuplement et formes de l'habitat dans les campagnes du Sud-Est de la France de l'Antiquité au Moyen Âge $\left(I V^{e}-V_{I I I}^{e}\right.$ S.)

Essai de synthèse., Gallia 64, Archéologie de la France, Paris, CNRS-Editions, 2007, p. 11-56; avec P. Rascalou, G. Colomer et A. Bergeret, « Genèse d'une villa carolingienne de l'arrière-pays biterrois $\left(\mathrm{VI}^{\mathrm{e}}-\mathrm{X}^{\mathrm{e}} \mathrm{S}\right.$. $)$ : la villa plaxano du cartulaire d'Aniane et la fouille des termes à Plaissan (Hérault) », Archéologie du Midi Médiéval 32-2014, Centre d'Archéologie Médiévale du Languedoc (CAML), 2016, p. 93-121. https://halshs. archives-ouvertes.fr/ halshs-01374131

\section{Hélène Wurmser}

est maître de conférences à l'université de Lyon 2 et directrice de l'IraA-Lyon (Institut de recherche sur l'architecture antique). Ses thèmes de recherche sont l'architecture et l'urbanisme grecs des époques hellénistiques et impériales. Elle a publié récemment : « L'habitat dans les Cyclades à l'époque impériale », in Topoi Orient-Occident 19, Lyon, MOM éditions, 2014, p. 309-323 ; avec J.-P. Guilhembet, « L'habitat familial urbain en Grèce et en Italie », in E. Parmentier et M. Coltelloni-Trannoy (coord.), Famille et société dans le monde grec, en Italie et à Rome du $v^{e}$ au II ${ }^{e}$ siècle avant J.-C., Pallas horssérie 02, Presses universitaires du Midi, 2017, p 227-258 ; «Nouvelles Recherches sur la Maison de Fourni à Délos », Bull. SFAC (XLVIII, 2016-2017), Revue Archéologique 65-1, 2018, p. 109-118.
Propos recueillis par Catherine Chauveau. Merci à Christiane Descombin. 

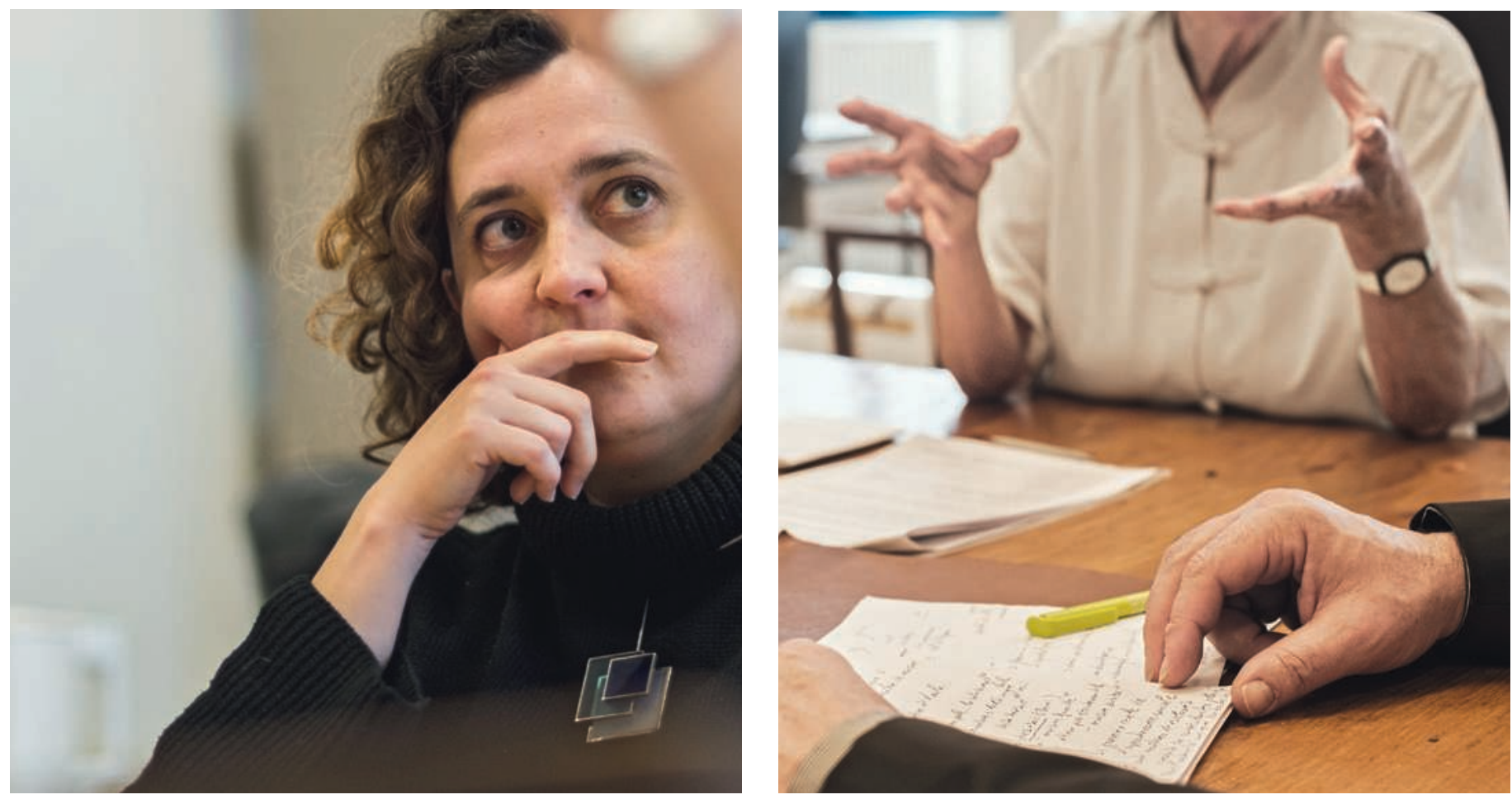

$\infty$
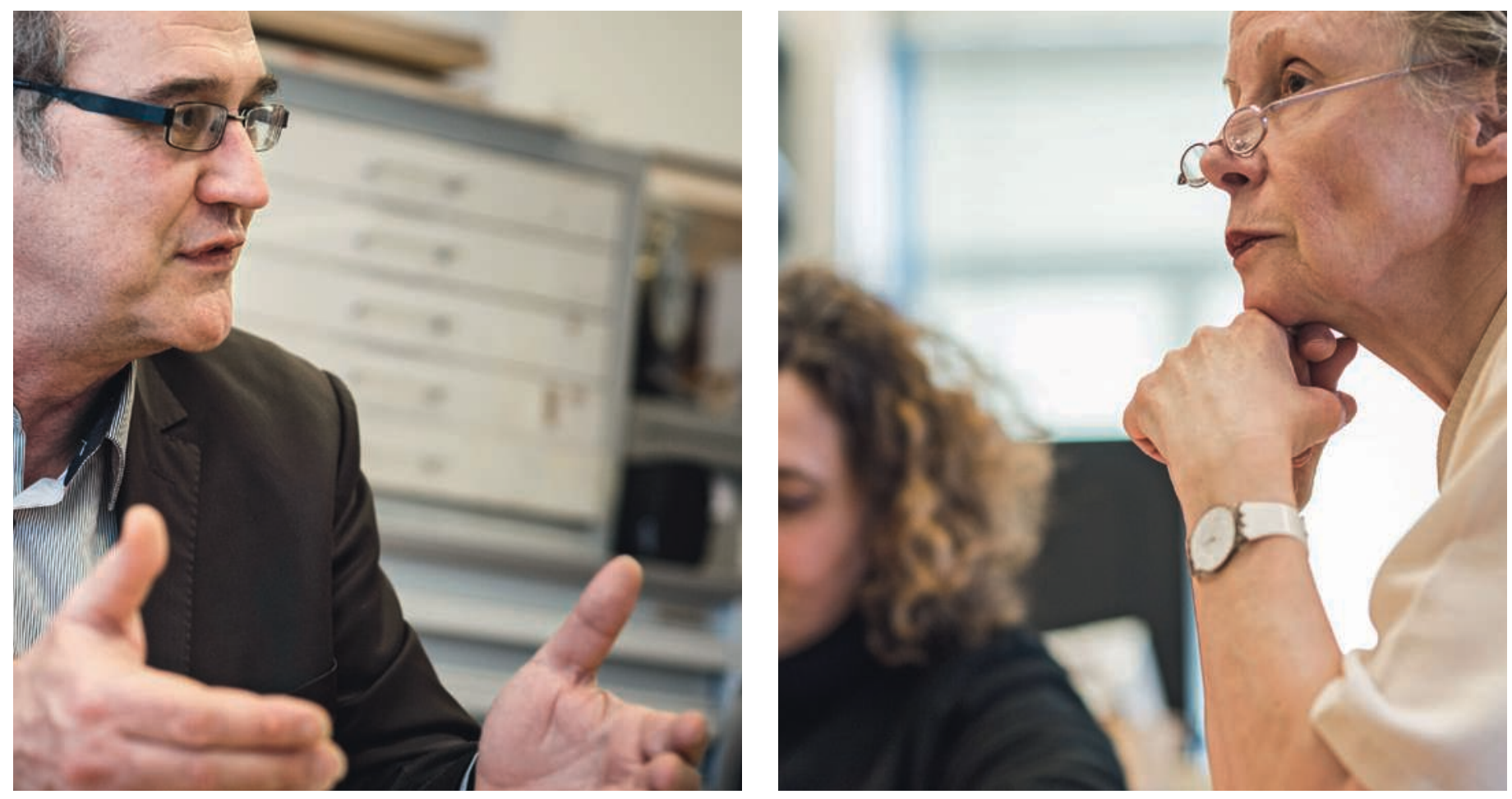

$\stackrel{\infty}{\stackrel{N}{N}}$

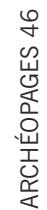


Laurent Schneider L'origine même du terme de maison est assez paradoxale, du point de vue du médiéviste. Elle est liée aux deux mots latins domus et mansus : domus, dérivé du dominus, le maître ; mansus, celui qui a l'obligation de « demeurer » en un lieu (qui deviendra le manant au Moyen Âge). Cela parle aux médiévistes : d'un côté, la maison qui va au-delà du bâti, qui regroupe une famille et des co-résidents placés sous l'autorité unique d'un patron; de l'autre, la maison des humbles et des dépendants, anciens esclaves casés et collectivités paysannes du Moyen Âge. Le plus intéressant est que ce ne soit pas domus qui ait donné le mot français de maison, mais mansus. On trouve les premières mentions de mansus à partir du $\mathrm{VI}^{\mathrm{e}}$ siècle et ce terme se fixe à l'époque carolingienne, à la fin du VIII ${ }^{\mathrm{e}}$ siècle, puis évolue en manse et enfin, dans le sud, en mas. Dans les autres langues latines que sont l'italien, l'espagnol et le portugais, la maison se dit casa, dérivé de casal, qui dans les textes du premier Moyen Âge désigne la petite maison paysanne, la case, la cabane. Mais que désigne domus dans un texte $\mathrm{du} \mathrm{IX}^{\mathrm{e}}$ siècle ? Le bâtiment d'habitation ? Les terres et les biens qui vont avec? Même incertitude pour le terme de villa, qui perdure aussi. On doit renoncer à mettre des formes derrière ces mots car les situations sont très diverses et l'on sent que ces deux termes sont liés aux conditions d'héritage des élites. Pour manse, le sens est tout autre. On nomme toujours le manse par le nom de son tenancier, le seigneur qui a la possession des terres. Le manant doit demeurer ; il ne peut être forcé par son patron à migrer sur une autre propriété ou d'une propriété à une autre. On voit bien, ici, que les textes renvoient à des catégories sociojuridiques et pas aux réalités matérielles sur lesquelles se basent nos études d'archéologue.

Anick Coudart La maison est aussi une entité sociale durable, parce qu'objectivée, pensée et perpétuée par un objet matériel vécu au quotidien, dont les structuration et codification spatiales produisent et reproduisent, dans le temps et pour chaque maisonnée, la vision partagée qu'une société a du monde. Les cultures que j'ai étudiées qu'elles soient des Amériques, d'Afrique, d'Asie ou d'Océanie - objectivent et perpétuent ainsi l'organisation et l'identité de leur société. La maison est un objet à la fois privé et universel pour la société qui la conçoit, la construit et l'habite.

Hélène Wurmser Oui, l'habiter et le rendre habitable correspondent à une révolution mentale de l'humanité dans la compréhension et le traitement des frontières entre soi et l'autre. Et ce sont bien les liens avec l'appropriation et le social, et non avec la technique ou la forme, qui nous font comprendre comment identifier ce qu'est la maison. La question de l'habitat met en jeu différentes approches scientifiques purement archéologiques (architecture, matériel, etc.) mais c'est aussi un sujet qui permet de confronter toutes sortes de sources. En grec ancien, le lieu où l'on habite se dit oikia, terme lié à la notion d'oikos qui désigne à la fois le fait de résider et celui de gérer : gérer les possessions de la maisonnée, l'état de la maison, les biens stockés... Cette notion essentielle de stockage a souvent été mise de côté en archéologie grecque, notamment en ce qui concerne les maisons urbaines. Elle est très peu documentée pour les fouilles anciennes car cet aspect n'a pas vraiment été envisagé. Aujourd'hui, il ne s'agit pas de se limiter aux entrepôts et aux amphores que l'on peut retrouver, mais d'intégrer dans la réflexion ce qui a disparu et n'a pas forcément laissé de traces : les coffres, les étagères, les boîtes, tout l'équipement de l'intendance... Quant à la maison rurale, elle se caractérise par l'emploi de nombreux mots qui désignent diverses « dépendances », selon les produits stockés et les types d'activité. Mais ces lieux aux noms spécifiques sont intégrés dans le bâtiment principal ou dans d'autres bâtiments, sans plan fixé.

LS C'est aussi vrai pour le Moyen Âge, comme pour les périodes antérieures : le lien entre unité d'habitation et unité d'exploitation est souvent manifeste. Qu'il y ait peu ou de nombreux bâtiments, on distingue les vestiges du bâtiment de résidence proprement dit et, autour, d'autres fonds de cabane qui généralement sont des annexes : ateliers, greniers, celliers, granges, silos... Mais la maison n'est pas différente des autres constructions.

HW En tout cas, le but est bien d'assurer la survie de la maisonnée, du minimum vital à la fructification des biens. Rien dans oikia ne détermine la forme ou les matériaux de l'édifice. Ce qui m'a fait regarder d'un œil critique les typologies établies pour les maisons grecques est cette croyance que le mode de fonctionnement se déduit de la forme. La répétition d'un plan voudrait dire, par exemple, que l'on est dans une société égalitaire, avec cette idée prégnante de la démocratie grecque qui rayonne partout. Or plusieurs sources montrent clairement que ce n'était pas le cas et Athènes elle-même en est le contre-exemple parfait! C'est pour cela que je suis sceptique envers l'usage du terme d'architecture domestique.

AC Mais « architecture domestique » est une notion utile pour qui étudie la structuration spatiale de la maison. La stabilité de la structuration de l'espace domestique s'observe dans toutes les sociétés, hiérarchisée ou non. Il en va ainsi des oppositions entre le féminin et le masculin, le propre et le sale, le haut et le bas, l'humide et le sec, le privé et la réception... Prenons des exemples actuels. Traditionnellement en Europe, le lieu des toilettes - le sale - et lieu de la toilette - le propre - sont séparé, même si aujourd'hui d'autres considérations prennent le pas (gain d'espace, réduction des coûts...) qui font que les toilettes sont de plus en plus installées dans la salle de bains. Au Japon, à la différence de l'Europe, on ne fait jamais sécher le linge dans la 


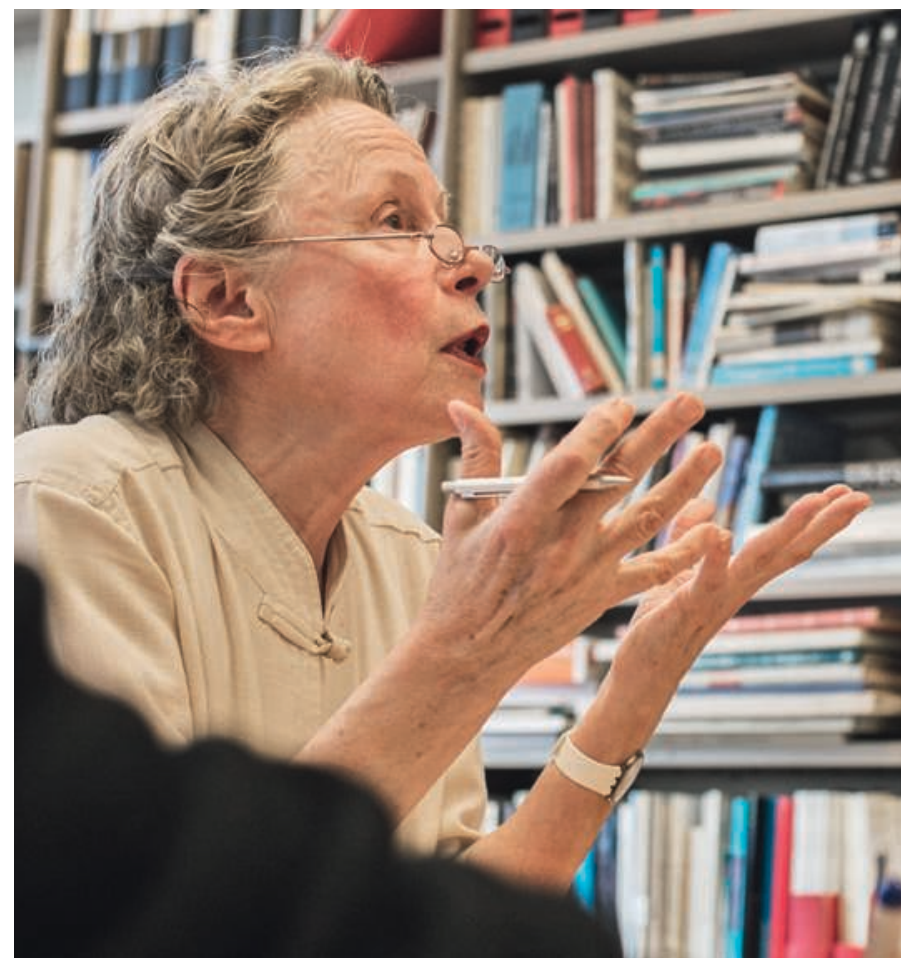

salle de bains parce que l'on ne mélange pas le sec et le mouillé. Regardons la maison de la civilisation néolithique de l'Europe centre-occidentale ${ }^{\mathbf{1}}$ (dite rubanée ou danubienne) ou celles, contemporaines, des Papous baruya, des Amérindiens navajos ou hopis. Si on compte le nombre d'options culturellement admises et utilisées pour la mise en place de certaines composantes de la maison (forme du toit, orientation, forme plan, partition intérieure...), on s'aperçoit que, bien souvent, un seul agencement a été mis en œuvre ou qu'une option a été utilisée dans $80 \%$ des habitations mais que deux ou trois autres types, souvent minoritaires, coexistent dans une même communauté. Dans la maison vit une famille restreinte ou élargie ou un lignage ou un clan, voire des alliés et des visiteurs temporaires. Les relations qu'entretiennent les membres de ces entités entraînent une structuration spatiale de l'espace. Par exemple, la maison traditionnelle des Baruya - circulaire et sur pilotis - est divisée en trois parties : entrée en chicane, espace féminin et espace masculin séparés par le foyer central. du $\mathrm{VI}^{\mathrm{e}}$ millénaire à la première $d u v^{e}$ millénaire.

\section{La stabilité de la} structuration de l'espace domestique s'observe dans toutes les sociétés, hiérarchisées ou non.

\author{
Anick Coudart
}

Dans ces terres hautes de Nouvelle-Guinée, il n'y a pas de terrain horizontal et les pentes sont fortes. L'espace réservé hommes se trouve au bas de la pente, là où les pilotis se doivent d'être plus hauts afin que le plancher soit horizontal. Les hommes sont ainsi symboliquement et physiquement en position dominante à l'égard de l'espace féminin qui jouxte l'entrée, construite en haut de pente - là où les pilotis sont plus courts. Dans ces villages existe une autre maison tout aussi traditionnelle, beaucoup plus grande : la maison où les adolescents vivent avec les hommes adultes qui doivent les initier pour devenir des hommes. C'est un autre mode de séparation entre masculin et féminin.

HW Les maisons des Grecs, à l'époque classique, peuvent avoir une salle des hommes, l'andron, qui sert de lieu de sociabilité entre « hommes libres » et de lieu de formation des jeunes hommes. C'est la seule pièce que l'on reconnaît sans trop de difficulté, par sa forme architecturale particulière. AC La partition de la maison baruya n'est pas sans rappeler celle des habitations du Néolithique rubané; plus de 2500 vestiges de maison ont été mis au jour entre la Pologne et la Normandie. Les maisons d'un village sont souvent de longueur différente (entre $8 \mathrm{~m}$ et plus de $20 \mathrm{~m}$ ) ; une plus grande construction (entre $25 \mathrm{~m}$ et $45 \mathrm{~m}$ ) du même type y figure, dont on pense, par rapprochement ethnographique, qu'elle servait à des usages collectifs extra-familiaux. Toutes ces maisons sont longues et quadrangulaires, spatialement rythmées par des travées de trois poteaux. Elles sont, le plus souvent, divisées en trois parties de l'entrée (dirigée vers le moyen Danube) vers l'arrière, 
avec une partie avant (un corridor précédé ou non d'un espace d'une ou deux travées de poteaux), un espace central de surface variable ; puis après un nouveau corridor et une partie arrière composée d'une ou deux ou trois (voire quatre) travées de longueur régulière. Il existe parfois un petit nombre d'éléments (façade, surface occupée, nombre de poteaux, grenier, sépulture d'enfant... surtout dans les régions occupées de longue date) qui n'entrent pas dans la typologie culturelle d'une population et qui sont liés à des choix individuels et/ou à un événement particulier. Ces différences sont autant de sources des transformations à venir. HW Oui, la réitération d'un certain nombre de formes a permis de créer des typologies, mais il nous faut en garder à l'esprit les limites, non négligeables, liées aux contingences dont vous parlez. Car il existe toujours pour un individu la possibilité de faire autrement. Aujourd'hui comme hier, on peut ne pas correspondre à son temps, à son lieu, à son milieu social : on peut ne pas être de son milieu en vivant dans un habitat communautaire ou dans un ancien château acheté pour rien; ne pas être de son lieu en équipant son appartement parisien à la japonaise; ne pas être de son temps, en n'utilisant pas certaines technologies. Si on transpose ceci pour l'époque impériale, de grands détenteurs du pouvoir ont fait construire des maisons de type méditerranéen (à l'italienne, à la grecque, etc.), c'est-à-dire des maisons à péristyle et à grandes cours, dans des contrées où l'on est en plein vent, où il neige de nombreux mois... Ils ont choisi de n'être pas de leur lieu. D'où le danger d'interpréter de travers des pseudo signes. En trouvant un bâtiment à péristyle, en Grèce, on pourrait conclure que c'est une maison type d'époque hellénistique. On oublierait alors que cette forme de bâti n'a été étudiée que dans certaines zones les plus connues, qu'elle est liée uniquement aux élites, et, enfin, que l'ordre architectural, les matériaux comme le marbre, les motifs décoratifs se retrouvent à l'identique dans l'architecture publique. Ces éléments ne sont donc pas distinctifs d'un habitat et l'architecture, en elle-même, ne suffit pas à interpréter. On arrive à reconnaître une maison uniquement quand on a la chance de pouvoir l'analyser comme ensemble archéologique : une architecture avec ce qu'elle contient. On trouvera des vestiges de ce qui tient de l'abri (se couvrir, dormir, préparer et consommer des repas...) et d'une structure familiale et sociale (stocker, séparer, recevoir, vénérer...). Même si on observe la réitération de certaines formes et des milieux déterminés, ce qui fait de la maison un chez-soi, quelle que soit sa forme, est avant tout une question d'appropriation.

LS La difficulté d'interprétation tient aussi de la réalité des durées d'occupation en contradiction avec des discours historiques énoncés comme des dogmes. Les historiens, et les archéologues à leur suite, ont cru longtemps à la fois dans la « parenthèse » romaine, dans l'existence de « déserts » aux débuts du Moyen Âge et dans la « naissance » des villages aux XI ${ }^{\mathrm{e}}$ et $\mathrm{XII}^{\mathrm{e}}$ siècles, quand des élites créent des lotissements avec parcelles à cultiver, pour attirer des colons moyennant des déductions fiscales. Ce que l'archéologie a apporté notamment pour le Moyen Âge, c'est la découverte d'un très grand nombre de bâtiments sur poteaux et la continuité des pôles d'occupation sur des siècles. Ces bâtiments de bois, de pisé, de terre, qui laissent des traces très difficilement lisibles, sont la persistance de traditions constructives protohistoriques qui ont traversé l'époque romaine. Si le pôle qu'était la villa a été abandonné, à cause de crises démographique, épidémique, économique etc., il y a continuité d'occupation dans de nouveaux pôles à quelques centaines de mètres alentour. Ces regroupements d'habitats et d'exploitation sont bien en place dès les $\mathrm{V}^{\mathrm{e}}-\mathrm{VI}^{\mathrm{e}}$ siècles, parfois avec leur église, sous les soi-disant nouveaux villages des $\mathrm{XI}^{\mathrm{e}}$ et $\mathrm{XII}^{\mathrm{e}}$ siècles. Cela donne des nuées de trous de poteau, difficiles à démêler chronologiquement, mais qui montrent à la fois les reconstructions et la permanence. Il nous a donc fallu revenir sur la question de la maison paysanne, réputée si fragile qu'elle avait disparu sans laisser de traces.

HW On peut s'interroger sur ce qui fait que l'on se fourvoie ainsi. Régulièrement, l'archéologue se dit surpris de ce qu'il trouve, contre toute attente. C'est que nos présupposés se fondent en particulier sur cet héritage quasi déférent par rapport à des textes, et sur cette sacro-sainte typologie formée par la pensée évolutionniste - comme si on allait continûment vers le mieux, vers le plus grand, vers le plus confortable. Ce sont de formidables fardeaux à certains égards. En entrant dans un logement actuel, on sait tous plus ou moins où se situera le salon par rapport à la chambre, lieu d'intimité pour nous, et faire la distinction entre ces deux pièces. Mais nous n'avons plus toutes les règles de fonctionnement tacite connu par les habitants d'une maison antique ou médiévale. Même si on peut se référer à des textes, on sait qu'ils ne documentent pas de la même façon tous les pans de la société. Quand les archéologues du $19^{\mathrm{e}}$ siècle sont partis à Délos, ou à Olynthe, avec sous le bras leur Vitruve et leur Économique de Xénophon, ils ont été fort dépités par les vestiges. En fins connaisseurs de la terminologie vitruvienne qui les encourageait à faire coïncider vocabulaire et réalité archéologique, ils ont parfois été tentés de forcer l'interprétation des vestiges. Ce qui a aussi faussé leur vision des choses, c'est l'extraordinaire état des maisons de Pompéi et d'Herculanum qui donnait l'impression que tout était sûr et identifiable. Et pourtant... Pénélope Allison ${ }^{2}$ a repris le travail sur les maisons bien fouillées à Pompéi, en recontextualisant le mobilier. Elle s'est rendue compte que, dans un certain nombre de cas, les interprétations 


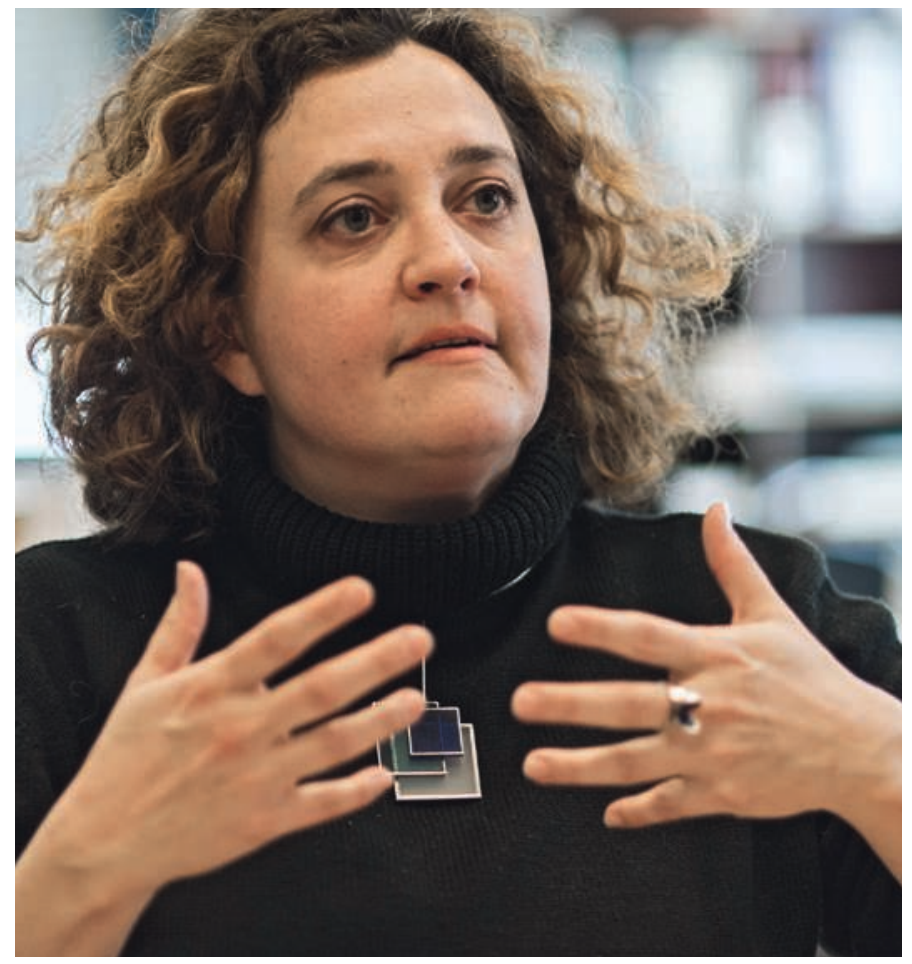

étaient fausses et que telle petite pièce sans fenêtre et avec une banquette maçonnée n'était pas une chambre, mais un espace de stockage de vaisselle, du moins dans son dernier état. Des générations d'archéologues antiquisants ont aussi tenté de formaliser, à l'imitation de Vitruve, des différences de matériaux entre le nord et le sud, entre rural et urbain etc. Mais là encore, on est forcé de constater que c'est un leurre. Il y a énormément d'élévations en terre dans les maisons grecques des périodes hellénistique et impériale y compris dans les plus belles maisons urbaines ! Les murs étant enduits à l'intérieur comme à l'extérieur, les élever en terre plutôt qu'en pierre permettait au moins d'économiser sur la construction.

LS Nous avions également pris en compte ces principes de Vitruve pour les périodes antiques et médiévales sur notre territoire. Et l'on voit bien,

\section{Il n'y a pas de matériaux, de techniques de construction ou de formes qui soient nécessairement spécifiques à l'habitat.}

Hélène Wurmser

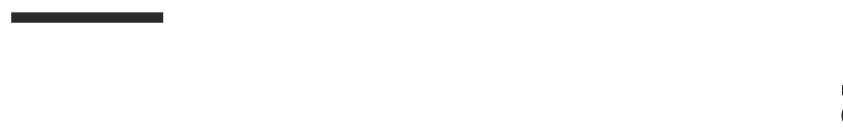

ont cherché aussi à déterminer les types régionaux de la maison française en s'appuyant notamment sur les matériaux. Mais ils ont renoncé à ces modèles trop rigides. Quant aux maisons de type traditionnel répertoriées par les ethnologues, elles sont assez récentes, datées, la plupart du temps entre les XVI ${ }^{\mathrm{e}}$ et $\mathrm{XIX}^{\mathrm{e}}$ siècles. Il est exceptionnel d'avoir des structures suffisamment bien conservées de maisons datant d'avant le XII ${ }^{\mathrm{e}}$ ou le XIII ${ }^{\mathrm{e}}$ siècle. D'après les premières fouilles de maisons médiévales, portant plutôt sur le bas Moyen Âge, on pense que c'est aux XIV ${ }^{\mathrm{e}}-\mathrm{XV}^{\mathrm{e}}$ siècles que se mettent en place de grands prototypes comme les zones à couverture de tuiles, les zones à couverture d'ardoises ou de chaume. S'il faut prendre en compte l'adaptation au climat et aux ressources disponibles, on a compris qu'il fallait moduler cette typologie selon d'autres critères économiques et sociaux (la gestion des carrières, le coût des transports de matériaux, les savoir-faire des artisans, la volonté de se démarquer des voisins ou de les copier...).

AC Les rapports au voisinage sont bien étudiés en ethnologie. Les deux tribus papoues que j'ai particulièrement suivies, les Baruya et les Ankave (qui appartiennent au même ensemble linguistique ${ }^{\mathbf{3}}$ ) ont exactement la même habitation traditionnelle. Ces groupes diffèrent cependant sur l'engagement social que représente la construction de la maison. Chez les Baruya, la maison est construite par les alliés et le lignage collectivement donc et en une journée. Chez les Ankave, elle est bâtie à deux : le propriétaire et un beau-frère ; la construction peut durer entre deux et quatre mois. Alors que les maisons 


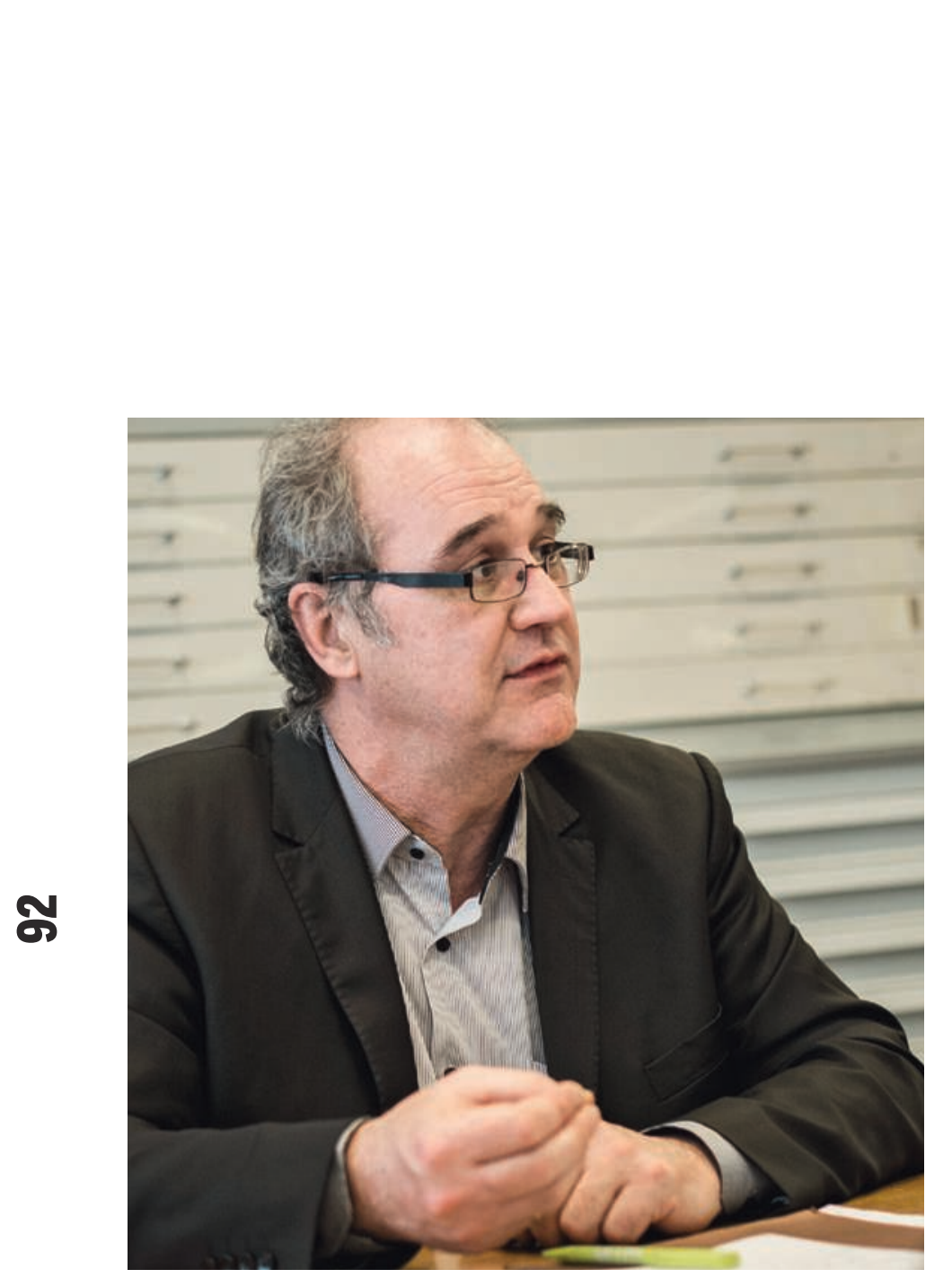

\title{
La persistance pendant l'époque romaine de traditions architecturales et de bâtiments protohistoriques est encore insuffisamment identifiée.
}

\author{
Laurent Schneider
}

principe qu'elle concerne d'abord les châteaux et les églises, donc qu'elle différencie socialement. On a recensé les bâtis en pierre à abside pour trouver les premières églises, avant de se rendre compte qu'il existait des églises en bois ! On est parfois incapable de discriminer une église d'une maison pour le haut Moyen Âge. En se fiant aux textes, on croyait les tombes situées dans et près des églises depuis l'Antiquité ; or ce type de cimetière, en fait, se met en place en Occident à partir de l'époque grégorienne, $\mathrm{au} \mathrm{XI}^{\mathrm{e}}$ siècle. L'inhumation à l'intérieur des maisons existe durant le haut Moyen Âge. Les édifices où l'on trouve des inhumations ne sont donc pas forcément des églises ! C'est passionnant, cette documentation que l'on crée tous les jours et qui permet de poser de nouvelles questions. Je reviens aux manants : des textes médiévaux mentionnent l'interdiction faite aux colons de démonter leurs maisons. Est-ce donc qu'elles étaient démontables et plutôt en bois? Est-ce que cette maison, sur la durée et son état de dégradation, peut être déplacée juste de quelques mètres? La maison de pierre, a priori, est plus difficile à déplacer; donc faite pour durer, pour se transmettre? L'usage de ces matériaux a-t-il à voir avec les modalités d'héritage? Dans le monde paysan, il y a forcément une transmission héréditaire par filiation. On peut diviser les terres, mais une trop forte division a des impacts négatifs sur la production agricole. Il s'ensuit forcément une émigration. Dans le cas d'une villa territoriale seigneuriale médiévale, on a des configurations différentes : la création de nouveaux domaines (le développement par les cadets de zones 
inexploitées), la mise en copropriété du domaine, la division du domaine (la multiplication des alleutiers, petits propriétaires libres). Au sud de la France, par exemple, on constate le choix de survie de la petite exploitation familiale, qui ne peut se maintenir dans la durée que si la démographie est constante. Pour le sud toujours, on explique par une croissance démographique les bouleversements complets des systèmes d'organisation des villages et de leurs maisons. L'espace se resserre, les bâtiments, d'emprise au sol réduite, deviennent mitoyens et se développent en hauteur. Les spécialisations des lieux vont se faire de manière verticale et non plus horizontale comme au haut Moyen Âge. Et cela renvoie à la question qui nous intéresse : celle de l'individualisation des logements. HW Question mise en perspective avec quelque chose que l'on a déjà évoqué : la continuité d'occupation, au moins sur plusieurs siècles. Les maisons d'époque impériale fouillées en Grèce ont régulièrement de trois à sept siècles d'occupation. Ce qui nous apparaît comme une entité architecturale peut s'avérer la maison non pas d'une famille mais de plusieurs. On sait cela par des textes juridiques. Parfois, c'est un partage entre héritiers, mais parfois ce sont des familles sans lien de parenté. Le temps long et cette partition évolutive des espaces font que les affectations des lieux changent - je vous suis entièrement sur le fait que notre raisonnement doit intégrer les étages, bien qu'ils soient très rarement conservés. À cette complexité s'ajoute la mobilité de certaines activités, comme la cuisine, le couchage, dont le mobilier est transportable (on n'a pas de fours maçonnés à Délos mais des réchauds mobiles, par exemple) : selon les circonstances, le moment de l'année, le temps qu'il fait, on va d'une pièce fermée, à la cour, à la terrasse... Ces modifications d'usage sur la durée peuvent être aussi dues à des mouvements de population assez importants. Délos est caractéristique parce que c'est une île qui devient complètement cosmopolite au milieu du $\mathrm{II}^{\mathrm{e}}$ siècle avant notre ère : elle est déclarée port-franc, exempté de taxes. Y affluent marchands et entrepositaires venant des rives orientales et méridionales de la Méditerranée : Italiens, Syriens, Égyptiens, Phéniciens, juifs.

Ils réoccupent un quartier ancien, le quartier du théâtre, et en construisent de nouveaux, les quartiers nord, mais selon les mêmes caractéristiques architecturales. Ces mélanges de populations n'ont pas conduit à une révolution complète dans les modes de construction. Toutes ces communautés se les sont appropriés en les adaptant. Je crois que « l'ethnicisation» du logement reste compliquée à déterminer. Les changements de formes ou de modes de constructions des maisons ne peuvent être systématiquement imputés à l'arrivée ou à l'influence d'une autre civilisation, d'une autre culture; il se produit aussi des évolutions culturelles au sein de sociétés qui relèvent d'une recherche d'efficacité, par exemple. En tout cas, les réponses ne sont jamais univoques. On se rend compte, qu'avec la maison, on touche à une des structures fondamentales du fonctionnement, comme l'alimentation, comme le vêtement. Ces grandes catégories connaissent des changements, plus ou moins rapides, de proche en proche, et sur des distances chronologiques qui peuvent être très longues. Mais, à mon avis, les modes de construction ont une pérennité exceptionnelle, jusqu'à ce qu'un événement majeur en bouleverse l'organisation. 